\title{
Tamizaje auditivo neonatal en pacientes de alto riesgo con otoemisiones acústicas: evaluación de resultados
}

\author{
Ricardo Silva Rueda \\ Otorrinolaringólogo \\ Profesor Universidad Militar Nueva Granada \\ Servicio de otorrinolaringologia del Hospital Militar Central \\ Email:silvarueda@gmail.com \\ Celular: 315335782 \\ Leonardo Elías Ordóñez Ordóñez \\ Otólogo-Otorrinolaringólogo . \\ Profesor Universidad Militar Nueva Granada \\ Servicio de otorrinolaringologia del Hospital Militar Central \\ Email: otoleor@gmail.com \\ Celular: 3112119428 \\ Diana Patricia Díaz Patiño \\ Residente III año de Otorrinolaringología \\ Hospital Militar Central - Universidad Militar Nueva Granada \\ Email: docdiaz7@yahoo.com \\ Celular: 3043633834 \\ Néstor Ricardo González Marín \\ Residente de Otorrinolaringología \\ Hospital Militar Central - Universidad Militar Nueva Granada \\ Email: nestorgm79@gmail.com Celular:3108064797
}

Claudia Ramírez

Fonoaudiologa - Audiología

Servicio de audiologia Hospital Militar Central

profesora ahdonorem

Email: jualcora@hotmail.com

Celular: 3003010046

\section{Dunia Paredes}

Fonoaudiologa - Audiología

Servicio de audiologia Hospital Militar Central

Email: duniaparedes@hotmail.com

Celular: 3204748073

\section{Silvia Raquel Rodríguez}

Fonoaudiologa - Audiología

Servicio de audiologia Hospital Militar Central

Docente asociada de la Universidad Nacional

Email: srrmontoya@gmail.com

Celular: 3188858019 


\section{Tamizaje auditivo neonatal en pacientes de alto riesgo con otoemisiones acústicas: evaluación de resultados}

\section{Resumen}

Objetivo: evaluar la efectividad del programa de tamizaje auditivo del Homic en pacientes de alto riesgo de hipoacusia neurosensorial, el cual está basado en la realización de otoemisiones acústicas

Diseño: estudio de cohorte retrospectiva, en el que se incluirán a los pacientes mayores de 12 meses de edad nacidos en el Hospital Militar central (HMC) entre enero de 2009 y diciembre de 2012; a quienes por condiciones de alto riesgo de hipoacusia neurosensorial se les realizó antes de los 6 meses de edad otoemisiones acústicas como prueba de tamizaje auditivo. A esta población, se le realizara diagnostico auditivo por medio del método diagnostico Gold Standart el cual es el "cross cheking”.

\section{Resultados:}

Se estudiaron los factores de riesgo para hipoacusia neurosensorial encontrando que el peso al nacer $\leq 1500$ gr. Se relacionó con hipoacusia en un $16 \%$. El $5.6 \%$ tiene antecedente familiar de hipoacusia neurosensorial en primer y segundo grado de consanguinidad. La infección congénita (TORCHS: toxoplasmosis, sífilis, rubéola, citomegalovirus, o herpes) como factor de riesgo se presentó en el $2.4 \%$ únicamente con la presencia de Toxoplasmosis, de los cuales 2 oídos presentaron cofosis.

\section{Conclusiones:}


La sensibilidad de las Otoemisiones acusticas para sospechar hipoacusia neurosensorial fue del $82.5 \%$. La especificidad estimada en nuestro estudio fue de $90.9 \%$

Neonatal hearing screening in high-risk patients with otoacoustic emissions: evaluation of results

\section{summary}

Objective: To evaluate the effectiveness of auditory screening program Central Military Hospital in patients at high risk of sensorineural hearing loss, which is based on the realization of otoacoustic emissions.

Design: Retrospective cohort study, in which over 12 months of age born patients in the Central Military Hospital (HOMIC) between January 2009 and December 2012 were included; who by conditions of high risk of sensorineural hearing loss was performed before 6 months of age otoacoustic emissions and hearing screening test. Total population was 125 patients. We evaluated each ear separately for a total of 250 measurements, this population was conducted auditory diagnosis through cross cheking diagnosis method were analyzed.

Results: Risk factors for sensorineural hearing loss were studied. We found that birth weight $\leq 1500 \mathrm{~g}$. It was associated with hearing loss by $16 \% .5 .6 \%$ have a family history of sensorineural hearing loss in first and second degree of consanguinity. Congenital infection (TORCHS: toxoplasmosis, syphilis, rubella, cytomegalovirus, or herpes) as a 
risk factor occurred in $2.4 \%$ only in the presence of Toxoplasmosis, of which 2 ears presented deafness.

Conclusions: The otoacoustic emissions are effective as hearing screening test in Military Central Hospital in patients at high risk of Sensorineural hearing loss.

The sensitivity of otoacoustic emissions to suspect sensorineural hearing loss was $82.5 \%$. The estimated specificity in our study was $90.9 \%$

Términos MESH

Otoemisiones Acústicas, hipoacusia neurosensorial, Factor de Alto riesgo Neonatal, Cross-check

Otoacoustic Emissions, sensorineural hearing loss, high risk factor Neonatal Cross-check 


\section{Introdución}

La hipoacusia se define como toda disminución de la agudeza auditiva que sobrepase los $27 \mathrm{db}$ en las frecuencias centrales del audiograma tonal.

Aproximadamente 1-5 de cada 1000 nacidos vivos estaría afectado con hipoacusia congénita bilateral severa a profunda, ${ }^{1,2}$ siendo esta cifra 10 veces superior (1-2\%) en las poblaciones con factores de riesgo. En Colombia la prevalencia es de 1.3\%; el $81.6 \%$ de las personas con discapacidad auditiva presenta pérdida auditiva parcial, el $18.4 \%$ presenta pérdida total y se presentan 3 casos de hipoacusia congénita por cada 1000 recién naidos vivos ${ }^{22}$.

El objetivo de la detección temprana de la hipoacusia es aprovechar al máximo las competencias lingüísticas y el desarrollo en la comunicación de los niños que la presenten.

Actualmente, en nuestro país, el promedio de edad en el cual se hace el diagnóstico sin contar con programas de detección precoz es alrededor de los 3 años. Es precisamente hasta esta edad el "periodo crítico" cuando existe en el ser humano la mayor plasticidad neuronal para hacer mayores sinapsis para el desarrollo de las vias auditivas y de la coteza auditiva en el lobulo temporal con la estimulacion y con ello se establecen las bases del desarrollo del lenguaje y de la comunicación.

Factores de riesgo que identifican a los recién nacidos, desde el nacimiento hasta los 28 días, que corren el riesgo de discapacidad auditiva neurosensorial son los siguientes (asha, 1991): 
1. Antecedentes familiares de hipoacusia neurosensorial (hns) congénita o de comienzo tardío.

2. Infecciones congénitas (torchs: toxoplasmosis, sífilis, rubéola, citomegalovirus, o herpes).

3. Anomalías craneofaciales, incluyendo anomalías morfológicas del pabellón auricular y el cae, filtrum ausente, e implantación baja del cabello.

4. Peso al nacer inferior a $1500 \mathrm{gr}$.

5. Incompatibilidad rh e hiperbilirrubinemia grave que requiera exanguineo transfusión.

6. Medicamentos ototóxicos (los aminoglucósidos utilizados por más de 5 días (ej.

Gentamicina, tobramicina, kanamicina, estreptomicina) y diuréticos de asa utilizados en combinación con aminoglucósidos, aines.

7. Meningitis bacteriana.

8. Hipoxia perinatal por puntuaciones de apgar de 0 a 3 a los 5 minutos o aquellos con dificultad para iniciar respiración espontánea por 10 minutos o aquellos con hipotonía persistente a las 2 horas de edad

9. Ventilación mecánica prolongada con una duración igual o superior a 5 días 10. Estigmas u otros hallazgos asociados con un síndrome asociados a hipoacusia ns (ej. Waardenburg o el síndrome de usher).

Factores que identifican a los recién nacidos, 29 días a 2 años, que están en riesgo de hns son los siguientes: 
1. Padres preocupados por el déficit auditivo o retraso en el lenguaje.

2. Meningitis bacteriana.

3. Factores de riesgo neonatales que pueden estar asociados con hns progresiva (ej. El citomegalovirus, la ventilación mecánica prolongada, y enfermedades heredadas).

4. Trauma de cabeza, especialmente con fractura longitudinal o transversal del hueso temporal.

5. Estigmas u otros hallazgos asociados con síndromes asociados a hns.

6. Medicamentos ototóxicos.

7. Niños con trastornos neurodegenerativos como la neurofibromatosis, epilepsia mioclónica, la enfermedad de werdnig-hoffmann, enfermedad de tay-sachs, la enfermedad de gaucher infantil, la enfermedad de niemann-pick, cualquier leucodistrofia metacromática, o cualquier neuropatía desmielinizante infantil.

8. Enfermedades infecciosas de la infancia que se sabe están asociados con la audición neurosensorial pérdida (por ejemplo, las paperas, el sarampión).

Si no existen factores de riesgo, los padres comienzan a sospechar de la existencia de una pérdida auditiva, entre los 8 y los 12 meses, si ésta es profunda debido a que perciben que el bebé no reacciona a ruidos fuertes, no se sobresalta, no vuelve la cabeza cuando se le llama, etc; y a edades mucho más avanzadas, 3 a 6 años si la pérdida es leve o moderada.

Existen medidas fisiológicas que se deben utilizar para detectar la pérdida auditiva en los recién nacidos y niños. Estas incluyen pruebas electrofisologicas como potenciales evocados, OAE, timpanometria, las cuales permiten obtener una idea de la actividad 
fisiológica subyacente de la función auditiva normal, y han sido utilizadas con éxito para tamizaje.

La evaluación del comportamiento de la audición requiere de técnicas especiales de prueba en los lactantes y niños pequeños para los cuales se realizan audiometrías infantiles. La técnica apropiada se basa en el grado del desarrollo psicomotor del niño. Si el niño tiene un desarrollo psicomotor normal, la edad cronológica se puede usar para la aplicación de ciertas técnicas. Pero a los niños que tienen retraso cognitivo y/o discapacidad física se debe ajustar la edad al nivel de sus habilidades.

\section{Otoemisiones acústicas (EOA)}

Las Emisiones Otoacústicas (EOA) son sonidos producidos por la actividad de las Celulas Ciliadas Externas en la coclea que se registran en el CAE; proporcionan una medida de la audición y su registro e interpretación no requiere mucho tiempo ni un equipo muy costoso, son de fácil implementación lo que la hace útil en el programa de tamizaje universal.

Las Celulas Ciliadas Externas del oido interno tienen la característica contraerse y por la presencia de actina y de generar energia mecanica la cual se obtiene en forma de sonido en el conducto auditivo externo mediante un micrófono sensible dentro de un

conjunto de una sonda que registra la respuesta coclear a los estímulos acústicos ${ }^{3}$ también tiene como caracteristic transformar el sonido que reciben del exterior en impulsos eléctricos, os cuales serán trasmitidos al cerebro a través del nervio Auditivo. 
Las EOA reflejan el estado del sistema auditivo periférico ya que son el resultado de la actividad fisiológica de las células ciliadas externas, por lo que su presencia se relaciona con un buen funcionamiento de los mecanismos cocleares activos. ${ }^{4,5}$

Las EOA están presentes en el 96-100\% de las personas con audición normal, y estan ausentes en pérdidas auditivas de más de $35 \mathrm{db}$, pero tambien estan ausentes cuando hay tampon de cerumen en el CAE o hay liquido en oido medio, o liquido en el CAE. Tienen una sensibilidad de $50 \%$ y una especificidad del $84 \%$.

Las OEA pueden sero Producto de Distosion cuando se realiza un estimulo con dos tonos puros simultaneos, $\mathrm{O}$ transcientes cuando se realiza una evocacion transitoria con cliks.

\section{Potenciales evocados auditivos del tronco cerebral (BERA)}

También conocida como B.E.R.A. (Brainstem Evoked Responses Audiometry), es la prueba objetiva más utilizada en la actualidad, se puede aplicar a niños de edades muy tempranas y es necesario que el sujeto se encuentre dormido o sedado, en reposo, con silencio y oscuridad. Son una poderosa herramienta para valoración objetiva de la audición.

Se obtienen a partir de electrodos de superficie que registran la actividad neuronal generada en la cóclea, nervio auditivo, y tronco cerebral en respuesta a los estímulos acústicos entregados a través de un auricular ${ }^{4}$ por conducción aérea, que produce información en las frecuencias de $2000-4000$ Hz. Se realiza con estimulo Click, y aparecen a los 10 milisegundos una serie de ondas picudas (cinco), como respuesta neurológica a la señal auditiva, que coinciden con el paso del estimulo por los 
diferentes núcleos nerviosos, donde se encuentran las sinapsis(Grafico 1) evaluado asi la actividad periferica de la via auditiva. Los parámetros más importantes a valorar son las latencias de las ondas I, III y V, especialmente la $\mathrm{V}$, dado en estas se realiza el relevo que la via uditiva hace en el coliculo inferior y su desaparición nos permite reconocer el umbral de audición del sujeto explorado. Los potenciales evocados auditivos nos permiten determinar la presencia o ausencia de hipoacusia, al igual que determinar el sitio de la lesion para un diagnostico diferencial.

A partir de los 18 meses pueden compararse con las del adulto. Los BERA tienen una sensibilidad del $100 \%$ y una especificidad del $98 \% .^{5}$

\section{Potenciales evocados auditivos estado estable frecuencia especifica. (PEAeeMF)}

Los PEAee permiten el uso de estímulos de estado estable tales como los tonos continuos con una amplitud modulada sinusoidalmente. Estos estímulos tienen un contenido espectral más circunscrito con un primer pico a nivel de la frecuencia portadora, otro a la frecuencia portadora más la frecuencia de modulación, y un tercero a la frecuencia portadora menos la frecuencia de modulación. Estas características de los tonos modulados los convierten en estímulos más específicos en cuanto a frecuencia. Se puede hacer un audiograma electrofisiologico en las frecuencias de 500, 1000,2000 y $4000 \mathrm{hz}$.

Su ventaja radica no solo en la especificidad de la frecuencia sino que por el espectro de frecuencia angosto permite diferenciar mejor entre perdidas auditivas severes y profundas. 


\section{Timpanometria}

La impedanciometría es un medio objetivo de medir la integridad y función del mecanismo auditivo periférico. A través de ella se puede determinar: la presión existente en el oído medio, la movilidad de la membrana timpánica, la función de la trompa de Eustaquio, la movilidad y continuidad de los huesecillos de la cadena osicular y los umbrales del reflejo acústico y su dinámica. El término impedancia se refiere a la resistencia ofrecida por un sistema al paso de una corriente energética a través de él y hace alusión a la energía rechazada, mientras que la facilidad con la que un sistema permite el paso de la energía se denomina admitancia y está relacionada con la energía que este acepta. Por definición la admitancia o compliáncia es lo opuesto de la impedancia.

La evolución de la distensibilidad, según las modificaciones de la presión, refleja de manera simultánea los siguientes factores: - La facilidad de movimiento de la cadena a través del tímpano; - El estado funcional propiamente dicho de la cadena de huesecillos; - El estado de la caja timpánica ${ }^{7,8}$.

El aparato utilizado se denomina "impedanciómetro" y la gráfica donde se reflejan los resultados "timpanograma". 
Los resultados de varios estudios son claros en mostrar que los niños diagnosticados y tratados tempranamente tienen mayores posibilidades de lograr una rehabilitación auditiva exitosa. La rehabilitación auditiva va de la mano de la adquisición del lenguaje hablado como principal forma de comunicación. Las implicaciones negativas para el individuo, y la sociedad, de una rehabilitación tardía son enormes

\section{Audiometrías infantiles}

Determina de forma cuantitativa los umbrales auditivos con tonos puros, teniendo en cuenta que el umbral se refiere a la mnima intensidad a la que un sonido es recibido. Permite cuantificar el grado de perdida auditiva, el tipo de frecuencia afectada y si la hipoacusia es conductiva, neurosensorial o mixta. Permite valorar tambine la via aerea la cual nos muestra funcio del oido medio, de la coclea, y tambien la vía osea, en la valoramos la funcion de la coclea eliminando el examen del CAE, la membrana timpanica y el odio medio.

Para niños menores de dos años se reliza una audiometria de observacion conductural en campo libre. En la que se dan estimulos para obtener respuestas comportamentales al estimulo acustico. Se entrena al niño para localizar un sonido. Este es reforzado por un estimulo visual,

Para niños marores de 2 años se puede realizar una audiometria condcionada, en la que el niño es entrenadao para que al oiro un estimulo acustico eecture una respuesta motora. 
Para los bebés desde el nacimiento hasta 5 o 6 meses de edad, la evaluación de la audición se basa en la observación de los cambios en el comportamiento secundarios a la estimulación auditiva.

A partir de esta edad, los lactantes comienzan a localizar el sonido para localizar al sonido en un plano lateral. Por esto la Audiometría de refuerzo visual (VRA) aprovecha la tendencia del niño a recurrir a una fuente de sonido. ${ }^{6}$

\section{Materiales y metodos}

\section{Cálculo del tamaño y selección de la muestra:}

Se seleccionaron todos los pacientes menores de 6 meses que se catalogaron como alto riesgo de hipoacusia neurosensorial dados por los factores de alto riesgo, a quienes se les realizo otoemisiones entre el 1 de enero de 2009 y 30 de Diciembre de 2012. Realizando un muestreo no aleatorio por conveniencia.

\section{Criterios de inclusión:}

- Niños menores de 6 meses de edad y aquellos nacidos entre el primero de enero de 2009 y el 30 de Diciembre de 2012 a quienes se les realizo el tamizaje auditivo para hipoacusia neurosensorial.

- Niños con factores de riesgo para presentar alto riesgo de hipoacusia neurosensorial.

\section{Criterios de exclusión}


- Niños que presenten patología del conducto auditivo externo, atresia aural o microtia.

- Niños cuya causa de hipoacusia neurosensorial se haya presentado a una edad mayor de 6 meses de edad. Por Ej trauma craneoencefálico, neuroinfección.

- Niños no ubicables.

\section{Metodologia}

Teniendo en cuenta la base de datos del servicio de Otorrinolaringología / audiología del Hospital Militar Central se llamaron a los padres de todos los pacientes a quienes se realizo el tamizaje auditivo, entre el 1 de enero de 2009 hasta el 30 de diciembe de 2012, para realizar los exámenes requeridos para hacer el diagnostico auditivo crosschecking basado en la toma de pruebas audiologicas objetivas y subjetivas para lo cual se realizo audiometría infantil, timpanometría con toma del reflejo estapedial, nuevas otoemisiones acústicas, y potenciales evocados de frecuencia específica por vía aérea y potenciales por vía ósea.

Además se realizo una valoración médica con el otologo quien realizo diagnostico audiológico teniendo en cuenta el principio del metodo del cross-check o verificacion cruzada el cual dice que una simple prueba audiologica no puee ser considerada valida sin que sea verificada por otra prueba tienendo en cuenta asì pruebas electrofisiologicas (BERA, PEAeeMF, OEA, Timpanometria) que miden funcion auditiva y cruzandolas con pruebas comportamentales (Audiometria, Logoaudiometria) que miden el umbral auditivo y evaluan el nivel de comunicación del 
paciente, para hacer el diagnostico positivo o negativo para hipoacusia neurosensorial uni o bilateral, y si es postivo, clasificandolo en grados según corresponde a Hipoacusia neurosensorial leve, moderada, severa o profunda. Ver flujograma 1.

Lo anterior se realizo previa aceptación y firma del consentimiento informado en el cual los padres aceptaron participar en el estudio.

\section{Mediciones e instrumentos a utilizar:}

Los exámenes requeridos para el diagnostico auditivo fueron realizados por las Audiologas y Fonoaudiologas del Hospital militar central. Se realizo una audiometría de observación conductual (BOA) en niños menores de 2 años en campo libre, $Y$ se realizo una audiometría condicionada en niños mayores de 2 años.

Se usó un audiómetro marca MAICO MA 42, Italia portátil de dos canales con dispositivo de audición maestro, que permite pruebas audiométricas de tono puro de voz y de campo libre.

La timpanometria y reflejos estapediales se tomaron con equipo marca MAICO MI44, Italia . Las pruebas realizadas en el modo de prueba timpanométrica miden la movilidad del oído medio, el reflejo acústico ipsilateral, el reflejo contralateral, la degeneración del reflejo y la trompa de Eustaquio. 
Las otoemisiones acústicas y Los potenciales evocados auditivos de frecuencia específica y de estado estable se realizaron con equipos marca interacoustic, con los pacientes trasnochados.

\section{Resultados}

De los pacientes con alto riesgo neonatal del Hospital Militar Central se realizó Otoemisiones acústicas (OEA) durante el periodo comprendido entre enero de 2009 y diciembre de 2012 a 235 pacientes de los cuales cumplieron criterios de inclusión 193 y de exclusión 42, por otro lado, el $35 \%$ de los niños restantes presento seguimiento incompleto por lo que no fueron incluidos en el estudio. Se analizaron 125 pacientes evaluando cada oído por separado para un total de 250 mediciones (Flujograma 2).

El rango de edad de la población incluida, en la que se realizó el estudio audiológico y valoración médica para diagnóstico de hipoacusia, fue entre 2 a 62 meses con una media de 23.8 meses, donde el $53 \%$ de los casos fueron de sexo femenino (Tabla 1).

Se estudiaron los factores de riesgo para hipoacusia neurosensorial encontrando que el peso al nacer $\leq 1500 \mathrm{gr}$. Se relacionó con hipoacusia en un 16\%. Dentro de la población estudiada el $5.6 \%$ tiene antecedente familiar de hipoacusia neurosensorial en primer y segundo grado de consanguinidad. La infección congénita (TORCHS: toxoplasmosis, sífilis, rubéola, citomegalovirus, o herpes) como factor de riesgo se presentó en el $2.4 \%$ únicamente con la presencia de Toxoplasmosis, de los cuales 2 oídos presentaron cofosis. (Tabla 2) 
La presencia de Estigmas u otros hallazgos asociados con síndromes asociados a hipoacusia neurosensorial como el Sindrome de Waardenburg o el síndrome de Usher no se detectaron en nuestro estudio, no obstante, se encontraron otros síndromes como el Mowat Wilson y Síndrome de Down, que en el caso de 8 oídos (3.6\%) se asociaron a hipoacusia.

La incompatibilidad $\mathrm{Rh}$ que requirió Isoinmunizacion se presentó solo en el $1.6 \%$ de los pacientes, de estos, el diagnóstico de hipoacusia Neurosensorial leve se observó en 1 paciente (2 oídos). La ventilación mecánica prolongada con una duración igual o superior a 5 días como otro factor de Riesgo para presentar hipoacusia se requirió en el $20.8 \%$ de los casos encontrando que solo 4 de estos tienen diagnóstico de hipoacusia. También estuvieron expuestos a medicamentos ototoxicos como la amikacina y la indometacina el $6.4 \%$ de las mediciones; dentro de ellos no se presentó ningún caso de hipoacusia.

Los Factores de riesgo que con menor frecuencia evidenciamos fueron, la presencia de enfermedades infecciosas de la infancia como el sarampión y la parotiditis cuya frecuencia de presentación fue del 0,8\%, y el antecedente de trauma cráneoencefalico en el momento del nacimiento o secundario a accidentes, especialmente asociado con la existencia de fractura longitudinal o transversal del hueso temporal el cual fue de $1.6 \%$. Otros factores de riesgo descritos pero que no encontramos en nuestra cohorte 
de pacientes fueron la existencia de Meningitis bacteriana y de trastornos neurodegenerativos.

El diagnostico auditivo por medio de la prueba "cross checking" nos permitió calcular la frecuencia de pacientes con hipoacusia Neurosensorial en la cohorte estudiada encontrando una proporción del 15.6\% (n:40) con el diagnostico. Por otro lado, se observó que el grado de hipoacusia neurosensorial más frecuente fue la cofosis. La proporción de pacientes con hipoacusia neurosensorial según el grado puede observarse en la figura 1.

Se evaluó la precisión diagnostica de las otoemisiones acústicas contrastándolas con el gold standart para el diagnostico (cross checking) a través de la sensibilidad, especificidad y valores predictivo positivo y negativo. Encontramos que La sensibilidad de la prueba para sospechar hipoacusia neurosensorial estimada en nuestro estudio fue del $82.5 \%$. Con una confianza del $95 \%$ podemos afirmar que la sensibilidad de la prueba se encuentra entre 69,47 - 95,53\%. La especificidad estimada en nuestro estudio para las otoemisiones acústicas fue de $90.9 \%$ de esta manera con una confianza del 95\% podríamos afirmar que la especificidad de la prueba diagnóstica se encuentra entre $86,83-95,07 \%$. Otros valores de precisión diagnostica según el grado de hipoacusia pueden observarse en la tabla 3.

\section{Discusión}

Como se menciono anteriormente, se estima que aproximadamente 1-5 de cada 1000 
nacidos vivos estaría afectado con hipoacusia congénita bilateral severa a profunda, 9,10 siendo esta cifra 10 veces superior (1-2\%) en las poblaciones con factores de riesgo. En nuestro estudio encontramos que la prevalencia de Hipoacusia neurosensorial en pacientes de alto riesgo neonatal fue de $15.6 \%$ Este resultado es acorde con la literatura mundial.

Las ototemisiones acústicas han sido la prueba de detección de elección porque son: rápidas y fácil de usar en un recién nacido; no son invasivas; y altamente sensibles y reproducibles ${ }^{11}$. Obtuvimos dentro de los resultados que la sensibilidad de la prueba para sospechar hipoacusia neurosensorial fue del $82.5 \%$ confirmando que las Otoemisiones son un buen método de tamizaje auditivo. Los falsos positivos que obtuvimos durante la realización del estudio que fueron del 19\% creemos que se deben a la presencia de líquido en oído medio, a una obstrucción del conducto auditivo externo y/o a una inmadurez de las células ciliadas externas. Pero repitiendo la prueba de OEAT se reducen los falsos positivos y mejora la eficacia diagnóstica de las OEAT lo cual observamos en $10 \%$ de los pacientes.

La principal desventaja de las otoemisiones, sin embargo, es que no es capaz de detectar la sordera retro coclear que los recién nacidos en situación de riesgo son susceptibles, pero este tipo de hipoacusia acontece raramente en neonatos ${ }^{12}$ aproximadamente en el $2.1 \%$ de los niños con cofosis según Tang et al ${ }^{13}$. Es por esto que los dos métodos recomendados en la actualidad, para la detección de la función auditiva en pacientes de alto riesgo incluyen la realización de emisiones otoacústicas y 
los potenciales auditivos de tallo cerebral ${ }^{14}$. Ambos métodos realizan estimaciones de la probabilidad de la función auditiva en los recién nacidos. En nuestro estudio solo se utilizo las otoemisiones acústicas pues es el método que se viene realizando como tamizaje auditivo neonatal en el Hospital Militar Central.

Identificamos la prevalencia de hipoacusia neurosensorial para cada uno de los factores de riesgo mencionados encontrando que la ventilación mecánica en los niños fue el factor de riesgo mas importante correspondiéndose con un $20 \%$, esta según Galambos y Despland ${ }^{15}$, provoca hipoxia en el sistema nervioso central y la posibilidad de deterioro de la audición afectando significativamente el segmento periférico de la vía auditiva. Martinez-Cruz et al. ${ }^{16}$ informó que estar durante varios días bajo ventilación mecánica y la estancia hospitalaria prolongada fueron dos factores encontrados de manera relevante en los recién nacidos con pérdida auditiva neurosensorial. Nuestra investigación mostro que el sometimiento de la población recién nacida a ventilación mecánica por mas de 5 días fue estadísticamente significativa para la presencia de hipoacusia la cual correspondió al $1.6 \%$ de los pacientes.

Esta descrito que el uso de ototoxicos con una concentración en rango terapéutico induce una perdida auditiva neurosensorial. Para algunos estudios es el factor de riesgo más común (33.13\%), Para otros estudios la pérdida auditiva neurosensorial se presenta solo en el $2.86 \%{ }^{17,18,19}$. En nuestra investigación no se presento ningún caso de hipoacusia secundaria al uso de ototoxicos como la gentamicina e indometacina; muy posiblemente debido a la pequeña población que requirió el uso de estos 
medicamentos siendo del $4,8 \%$ y $1.6 \%$ respectivamente.

Ohl et al. Comparo recién nacidos con un factor de riesgo y recién nacidos con dos o más factores de riesgo y mostro que los niños con dos o más factores de riesgo tuvieron con mayor frecuencia diagnostico de hipoacusia neurosensorial siendo del $6.1 \%$ comparada con la de los recién nacidos con un solo factor de riesgo que fue del $1,6 \%{ }^{20} \sin$ embargo este no fue un objetivo de nuestro estudio, pero podría ser un campo de investigación futura a realizar en el Hospital militar central.

Según Ireneusz Bielecki en 2011 et al $^{19}$, durante su estudio encontraron un grupo grande de niños con pérdida auditiva neurosensorial que no estaba expuesto a ningún factor de riesgo conocido, lo que indica la importancia de realizar la prueba de tamizaje auditivo neonatal de manera universal. Lo mismo recomienda Declau et al en 1997. ${ }^{21}$ quien describe que un total de $55,8 \%$ de los niños con pérdida auditiva no tenía factores de riesgo en absoluto. Por lo anterior, recomendamos realizar el tamizaje auditivo neonatal a todos los recién nacidos, y no limitarnos a los neonatos con indicadores de alto riesgo para presentar hipoacusia, para poder así detectar precozmente un mayor número de niños con déficits auditivos.

Por ultimo, reconocemos que el tamizaje auditivo neonatal se realiza en nuestra institución únicamente en pacientes de alto riesgo para hipoacusia, siendo esta una pequeña población con la que también tuvimos inconvenientes en cuanto al ingreso de los niños al estudio. Una limitación de nuestro estudio fue que las personas que realizaron los estudios no eran ciegas al resultado de las pruebas de tamizaje debido a 
que se encontraban en las bases de datos.

Recomendamos realizar el tamizaje auditivo neonatal a todos los recién nacidos en el hospital militar para realizar detección temprana y tratamiento adecuado en los niños con perdida auditiva para aprovechar al máximo las competencias lingüísticas y el desarrollo en la comunicación de los niños que la presentan, debido a que estos niños se quedan atrasados con respecto a sus compañeros normoyentes en cuanto al desarrollo de la comunicación, la lectura y el desarrollo social-emocional.

\section{CONCLUSIONES}

Encontramos que las OEA son efectivas como prueba de tamizaje auditivo en el HOMIC en pacientes de alto riesgo de hipoacusia Neurosensorial.

La hipoacusia neurosensorial se presenta de manera similar en pacientes de ambos sexos, siendo del $53 \%$ en niñas y del $46-4 \%$ en niños del hospital militar central, con una prevalencia del $15.2 \%$.

La sensibilidad de las otoemisiones acústicas para diagnosticar hipoacusia neurosensorial en pacientes recién nacidos y menores de 6 meses con factores de alto riesgo de hipoacusia neurosensorial es del $82.5 \%$ con una especificidad del $90.9 \%$. 


\section{Referencias}

1. Universal newborn hearing screening, summary of evidence. Jama 2001, 286. Ferrer eduardo, marulanda m.d, tobón gloria, arredondo md, guerra lisandro $\mathrm{md}$.

2. Evaluación de la hipoacusia neurosensorial en el neonato. Acta de otorrinolaringología y cirugía de cabeza y cuello. 2004. Vol.

3. Year 2007 Position Statement: Principles and Guidelines for Early Hearing Detection and Intervention Programs, Joint Committee on Infant Hearing, Pediatrics 2007;120;898-921g, DOI: 10.1542/peds.2007-2333.

4. Archivos de Pediatría del Uruguay 2008; 79. 315-319.

5. Kemp DT. Stimulated acoustic emissions from within the human auditory system. J Acoustic Soc Am 1978; 74: 1386-91.

6. Audiologic assessment of children with suspected hearing loss. Otolaryngology Clinics of North America 35 (2002) 711-732.

7. Lic Mariana Maggio De Maggi Fonoaudióloga. La impedanciometría: Consideraciones Pediátricas.

8. Canadian Working Group on Chilhood Hearing. Early Hearing and Communication Development: Canadian Working Group on Childhood Hearing (CWGCH) Resource Document. Ottawa: Minister of Public Works and Govermment Services. Canada, 2005.

9. Anna M. H. Korver, MD, PhD Saskia Konings, MD Friedo W. Dekker, PhD Mieke Beers, PhD, Capi C. Wever, MD, PhD, Johan H. M. Frijns, MD, PhD Anne M. Oudesluys-Murphy, MB, PhD. Newborn Hearing Screening vs Later 
Hearing Screening and Developmental Outcomes in Children With Permanent Childhood Hearing Impairment. AMA, October 20, 2010—Vol 304, No. 15

10. Ferrer Eduardo, Marulanda M.D, Tobon Gloria, Arredondo MD, Guerra Lisandro MD. Evaluación de la hipoacusia neurosensorial en el neonato. Acta de otorrinolaringología y cirugía de cabeza y cuello. 2004. Vol.

11.P. Bonfils, P. Marie, P. Narcy, Qu'attendre des oto-e'missions acoustiques? Ann. Otolaryngol. 106 (1989) 290-293.

12. Ch. Ohl, L. Dornier, C. Czajka, J. -C. Chobat, L. Tavernier, Newborn hearing screening on infants at risk, Int. J. Pediatr. Otorhinolaryngol. 73 (2009) 16911695.

13.P. Tang, B. McPherson, K.C. Yuen, L.L. Wong, J.S. Lee, Auditory neuropathy/ auditory dys-synchrony in school children with hearing loss: frequency of occurrence, Int. J. Pediatr. Otorhinolaryngol. 68 (2004) 175-183.

14. Erenberg, J. Lemons, C. Sia, D. Trunkel, P. Ziring, New- born and infant hearing loss: detection and intervention. American Academy of Pediatrics. Task Force on Newborn and Infant Hearing, Pediatrics 103 (1999) 527—530.

15. Galambos, P.A. Despland, n, Pediatr. Res. 14 (1980) 159-163.

16. C.F. Martinez-Cruz, A. Poblano, L.A. Fernandez-Carrocera, Risk factors with sen- sorineural hearing loss in infants at the Neonatal Intensive Care Unit: 15year experience at the National Institute of Perinatology (Mexico City), Arch. Med. Res. 39 (2008) 686-694.

17. M. de Hoog, B. van Zanten, W.C. Hop, E. Overbosch, R.N. Weisglas-Kuperus, J.N. van den Anker, Newborn hearing screening: tobramycin and vancomycin are not risk 
factors for hearing loss, J. Pediatr. 142 (2003) 41-46.

18.P. van Dommelen, A.D. Mohangoo, P.H. Verkerk, C.P.B. van der Ploeg, H.L.M. van Straaten, Risk factors for hearing loss in infants treated in different Neonatal Intensive Care Units, Acta Paediatr. 93 (2010) 344-349.

19. Ireneusz Bielecki, Anna Horbulewicz, Teresa Wolan irsk factors associated with hearing loss in infants: An analysis of 5282 referred neonates. International Journal of Pediatric Otorhinolaryngology 75 (2011) 925-930.

20. F. Declau, A. Boudewyns, J. Van den Ende, A. Peeters, P. van den Heyning, Etiologic and audiologic evaluations after Universal Neonatal Hearing Screening: analysis of 170 referred neonates, Pediatrics 121 (2008) 1119-1126.

21. Cox LC. Otoacoustic emissions as a screening tool for sensorineural hearing loss. J Pediatr 1997; 130:685-686.

22. Hernández W, González J, Bernal L. Tamización universal auditiva neonatal: Acta de la reunión de equipo Medicina familiar [En Línea]. Departamento de medicina preventiva y social. Pontificia Universidad Javeriana. Octubre 2007.

Grafico 1. ONDAS DE MORFOLOGIA DE VIA AUDITIVA

\begin{tabular}{|l|l|l|}
\hline Onda & Latencia & Origen \\
\hline I & $1.5 \mathrm{msg}$ & Nervio acústico \\
\hline II & $2.5 \mathrm{msg}$ & Protuberancia \\
\hline III & $3.5 \mathrm{msg}$ & $\begin{array}{l}\text { Complejo olivar } \\
\text { superior }\end{array}$ \\
\hline IV & $4.5 \mathrm{msg}$ & Coliculos inferiores \\
\hline V & $5.5 \mathrm{msg}$ & Mesencéfalo \\
\hline
\end{tabular}


Flujograma 1. Protocolo de evaluación de tamizaje auditivo neonatal en Hospital Militar Central

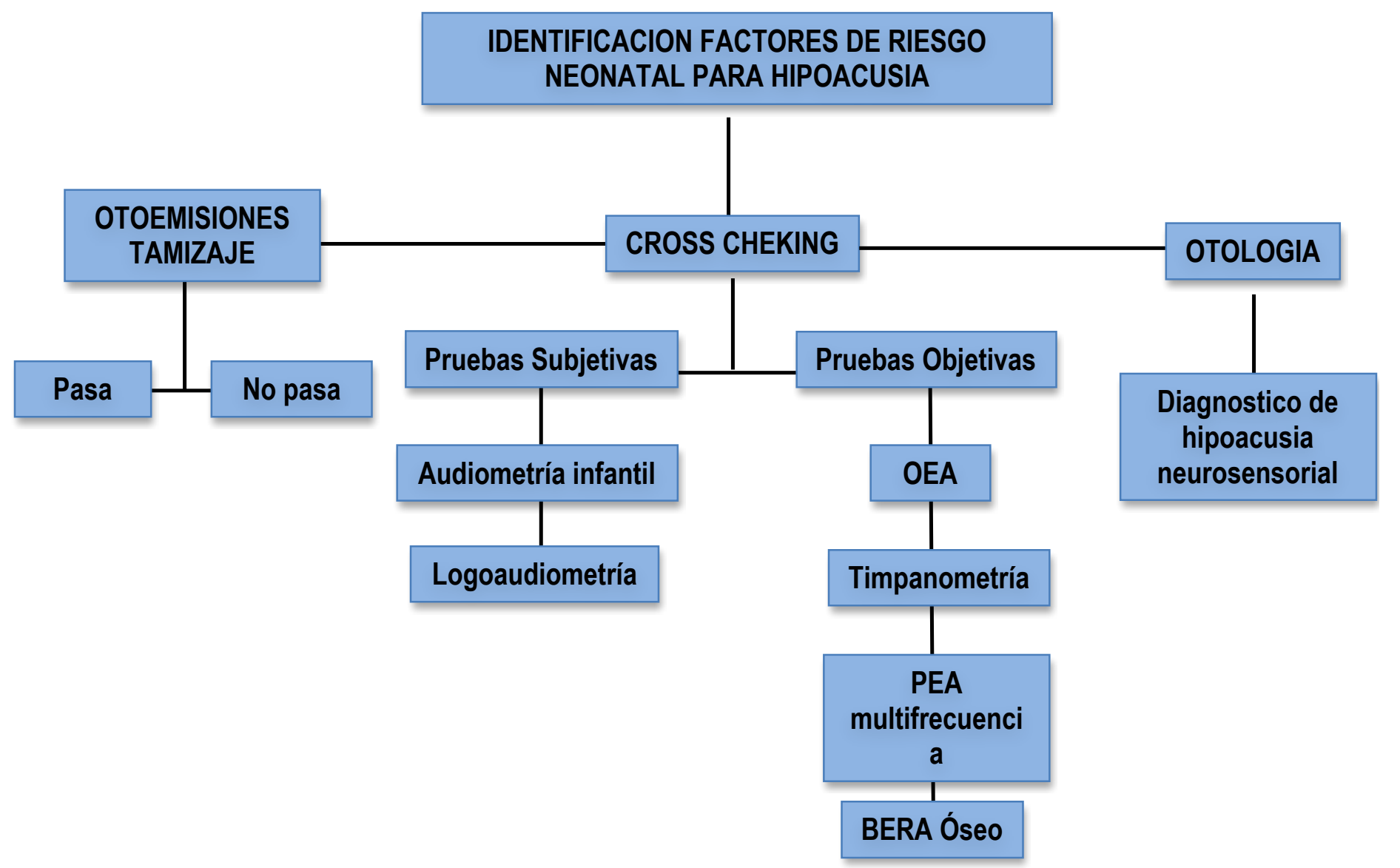


Flujograma 2. Reclutamiento de pacientes con alto riesgo neonatal en Hospital Militar Central

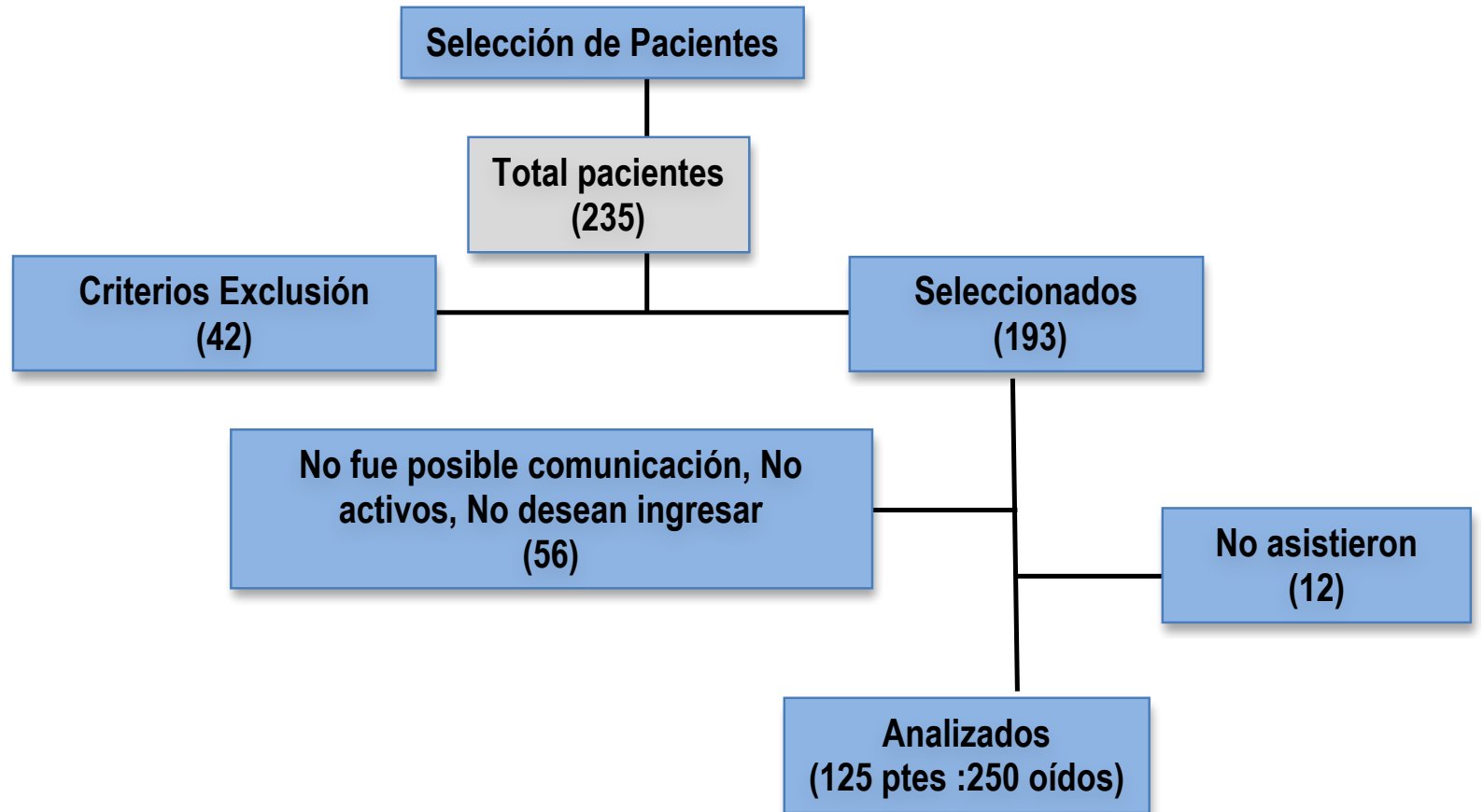

\begin{tabular}{|c|c|c|c|}
\hline \multicolumn{4}{|c|}{$\begin{array}{l}\text { TABLA 1. CARACTERIZACION DE DATOS DEMOGRAFICOS DE } \\
\text { PACIENTES CON ALTO RIESGO NEONATAL EN HOSPITAL } \\
\text { MILITAR CENTRAL }\end{array}$} \\
\hline & Media & $\pm D E$ & Rango \\
\hline Edad tamizaje & $4.03 \mathrm{~m}$ & $4.85 \mathrm{~m}$ & $1 m-26 m$ \\
\hline Edad Dx audiológico & $23.8 \mathrm{~m}$ & $15.27 \mathrm{~m}$ & $2 m-62 m$ \\
\hline & $\%$ & $(n=250)$ & [IC 95\%] \\
\hline $\begin{array}{l}\text { Femenino } \\
\text { Masculino }\end{array}$ & $\begin{array}{c}53 \% \\
46.4 \% \\
\end{array}$ & $\begin{array}{l}(n: 134) \\
(n: 116)\end{array}$ & $\begin{array}{l}{[46.8-59.2 \%]} \\
{[40.2-52.6 \%]}\end{array}$ \\
\hline $\begin{array}{ll}\text { Fuerza } & \text { Ejercito } \\
& \text { Armada } \\
& \text { FAC } \\
& \text { HOMIC }\end{array}$ & $\begin{array}{l}80.4 \% \\
11.6 \% \\
6.9 \% \\
0.8 \%\end{array}$ & $\begin{array}{l}(\mathrm{n}: 201) \\
(\mathrm{n}: 28) \\
(\mathrm{n}: 17) \\
(\mathrm{n}: 2)\end{array}$ & $\begin{array}{c}75.5-85.3 \%] \\
{[7.6-19.6 \%]} \\
{[3.8-10 \%]} \\
{[-0.3-1.9 \%]}\end{array}$ \\
\hline $\begin{array}{ll}\text { Peso al nacer } & <1500 \\
& >1500\end{array}$ & $\begin{array}{l}19.2 \% \\
80.4 \%\end{array}$ & $\begin{array}{c}(n: 48) \\
(n: 198)\end{array}$ & $\begin{array}{l}{[14.3-24.1 \%]} \\
{[74.5-85.3 \%]}\end{array}$ \\
\hline
\end{tabular}




\begin{tabular}{|c|c|c|c|c|c|}
\hline & & & $\%$ & $(n=250)$ & [IC 95\%] \\
\hline $\begin{array}{l}\text { Antecedente fa } \\
\text { de hipoacusia }\end{array}$ & iliar & $\begin{array}{l}\mathrm{Si} \\
\text { No }\end{array}$ & $\begin{array}{l}5.6 \% \\
94.4 \% \\
\end{array}$ & $\begin{array}{c}(\mathrm{n}: 14) \\
(\mathrm{n}: 236) \\
\end{array}$ & $\begin{array}{c}{[2.7-8.4 \%]} \\
{[91.5-97.2 \%]}\end{array}$ \\
\hline TORCH & $\overline{T o x c}$ & plasmosis & $2.4 \%$ & $(n: 6)$ & {$[0.5-4.2 \%]$} \\
\hline Isoinmunizació & & & $1.6 \%$ & $(n: 4)$ & {$[0.04-3.1 \%]$} \\
\hline Exanguineo-tra & sfusi & & $2.4 \%$ & $(n: 6)$ & {$[0.5-4.2 \%]$} \\
\hline Ototoxicos & $\begin{array}{l}\text { Amil } \\
\text { Indo }\end{array}$ & $\begin{array}{l}\text { acina } \\
\text { metacina }\end{array}$ & $\begin{array}{l}4.8 \% \\
1.6 \%\end{array}$ & $\begin{array}{c}(\mathrm{n}: 12) \\
(\mathrm{n}: 4)\end{array}$ & $\begin{array}{c}{[2.1-7.4 \%]} \\
{[0.04-3.1 \%]}\end{array}$ \\
\hline Meningitis & & & & & 0 \\
\hline $\begin{array}{l}\text { Ventilación } \\
\text { Mecánica }\end{array}$ & $\begin{array}{l}\text { Si } \\
\text { No }\end{array}$ & $\begin{array}{l}<5 \text { días } \\
>5 \text { días }\end{array}$ & $\begin{array}{c}8 \% \\
20.8 \% \\
71.2 \% \\
\end{array}$ & $\begin{array}{c}(n: 20) \\
(n: 52) \\
(n: 178)\end{array}$ & $\begin{array}{c}{[4.6-11.3 \%]} \\
{[15.7-25.8 \%]} \\
{[65.5-76.8 \%]}\end{array}$ \\
\hline Síndromes & $\begin{array}{l}\text { Otro } \\
\text { No t }\end{array}$ & ene & $\begin{array}{c}8.8 \% \\
91.2 \%\end{array}$ & $\begin{array}{c}(n: 22) \\
(n: 228)\end{array}$ & $\begin{array}{l}{[5.31-12.3 \%]} \\
{[87,7-94.7 \%]}\end{array}$ \\
\hline $\begin{array}{l}\text { Fractura del } \\
\text { Peñasco }\end{array}$ & $\begin{array}{l}\mathrm{Si} \\
\text { No }\end{array}$ & & $\begin{array}{c}1.6 \% \\
98.4 \%\end{array}$ & $\begin{array}{c}(n: 4) \\
(n: 246)\end{array}$ & $\begin{array}{c}{[0.04-3.1 \%]} \\
{[96.8-99 \%]}\end{array}$ \\
\hline T. Neurodegen & rativo & & & & 0 \\
\hline Paramixovirus & $\begin{array}{l}\text { Sara } \\
\text { Parc } \\
\text { No }\end{array}$ & $\begin{array}{l}\text { mpión } \\
\text { tiditis }\end{array}$ & $\begin{array}{c}0.8 \% \\
0.8 \% \\
98.4 \%\end{array}$ & $\begin{array}{c}(n: 2) \\
(n: 2) \\
(n: 146)\end{array}$ & $\begin{array}{l}{[-0.3-1.9 \%]} \\
{[-0.3-1.9 \%]} \\
{[96.8-99 \%]}\end{array}$ \\
\hline
\end{tabular}

Frecuencias y porcentajes se reportan por número de oídos estudiados.

[IC 95\%] Intervalo de confianza 95\%

\begin{tabular}{|c|c|c|c|c|c|c|c|c|}
\hline & $\begin{array}{l}\text { Drueb } \\
\text { oss C }\end{array}$ & $\begin{array}{l}\text { e Oro } \\
\text { king) }\end{array}$ & $\mathbf{S}$ & $\mathbf{E}$ & [IC 95\%] & VVP & VPN & $\mathbf{P}$ \\
\hline \multirow{2}{*}{$\begin{array}{c}\text { Prueba en } \\
\text { estudio } \\
\text { (Otoemisiones } \\
\text { acústicas) }\end{array}$} & $\begin{array}{l}\text { VP } \\
33\end{array}$ & $\begin{array}{l}\text { FP } \\
19\end{array}$ & \multirow[t]{2}{*}{$82.5 \%$} & & & \multirow{2}{*}{$63.4 \%$} & \multirow{2}{*}{$49.4 \%$} & \multirow[t]{2}{*}{$16 \%$} \\
\hline & $\begin{array}{c}\mathrm{FN} \\
7\end{array}$ & $\begin{array}{l}\text { VN } \\
191 \\
\end{array}$ & & $90.9 \%$ & {$[86,83-95,07 \%]$} & & & \\
\hline & \multicolumn{2}{|c|}{ HNS Leve } & $\mathbf{S}$ & E & [IC 95\%] & VVP & VPN & $\mathbf{P}$ \\
\hline \multirow{2}{*}{$\begin{array}{l}\text { Otoemisiones } \\
\text { acústicas }\end{array}$} & VP & FP & \multirow[t]{2}{*}{$37.5 \%$} & & {$[0-77,3 \%]$} & \multirow[b]{2}{*}{$12.5 \%$} & \multirow[b]{2}{*}{$97.4 \%$} & \multirow[b]{2}{*}{$3.6 \%$} \\
\hline & $\begin{array}{c}\mathrm{FN} \\
5 \\
\end{array}$ & $\begin{array}{l}\text { VN } \\
191\end{array}$ & & $90 \%$ & {$[85,8-94,3 \%]$} & & & \\
\hline & \multicolumn{2}{|c|}{$\begin{array}{c}\text { HNS } \\
\text { Moderada }\end{array}$} & $\mathbf{S}$ & E & [IC 95\%] & VVP & VPN & $\mathbf{P}$ \\
\hline \multirow{3}{*}{$\begin{array}{l}\text { Otoemisiones } \\
\text { acústicas }\end{array}$} & $\mathrm{VP}$ & $\mathrm{FP}$ & $100 \%$ & & {$[75-100 \%]$} & \multirow{3}{*}{$8.7 \%$} & \multirow{3}{*}{$99.7 \%$} & \multirow{3}{*}{$0.9 \%$} \\
\hline & 2 & 21 & & & & & & \\
\hline & $\begin{array}{c}\mathrm{FN} \\
0 \\
\end{array}$ & $\begin{array}{l}\text { VN } \\
191 \\
\end{array}$ & & $90 \%$ & {$[85,8-94,3 \%]$} & & & \\
\hline & \multicolumn{2}{|c|}{ HNS Severa } & $\mathbf{S}$ & E & [IC 95\%] & VVP & VPN & $\mathbf{P}$ \\
\hline \multirow{3}{*}{$\begin{array}{l}\text { Otoemisiones } \\
\text { acústicas }\end{array}$} & VP & FP & $100 \%$ & & {$[93,7-100 \%]$} & \multirow{3}{*}{$27.5 \%$} & \multirow{3}{*}{$100 \%$} & \multirow{3}{*}{$3.6 \%$} \\
\hline & 8 & 21 & & & & & & \\
\hline & $\begin{array}{c}\mathrm{FN} \\
0\end{array}$ & $\begin{array}{l}\text { VN } \\
191\end{array}$ & & $90 \%$ & {$[85,8-94,3 \%]$} & & & \\
\hline
\end{tabular}




\begin{tabular}{|c|c|c|c|c|c|c|c|c|}
\hline & & da & $\mathbf{S}$ & $E$ & [IC 95\%] & VVP & VPN & $\mathbf{P}$ \\
\hline \multirow{3}{*}{$\begin{array}{l}\text { Otoemisiones } \\
\text { acústicas }\end{array}$} & VP & FP & \multirow[t]{3}{*}{$100 \%$} & \multirow[b]{3}{*}{$90 \%$} & {$[94,4-100 \%]$} & \multirow{3}{*}{$30 \%$} & \multirow{3}{*}{$100 \%$} & \multirow{3}{*}{$4 \%$} \\
\hline & 9 & 21 & & & & & & \\
\hline & $\begin{array}{c}\text { FN } \\
0\end{array}$ & $\begin{array}{c}\text { VN } \\
191\end{array}$ & & & {$[85,8-94,3 \%]$} & & & \\
\hline & \multicolumn{2}{|c|}{ Cofosis } & $\mathbf{S}$ & $E$ & [IC 95\%] & VVP & VPN & $\mathbf{P}$ \\
\hline \multirow{3}{*}{$\begin{array}{l}\text { Otoemisiones } \\
\text { acústicas }\end{array}$} & VP & FP & \multirow[t]{3}{*}{$84.6 \%$} & \multirow[b]{3}{*}{$90 \%$} & {$[61-100 \%]$} & \multirow{3}{*}{$34 \%$} & \multirow{3}{*}{$98 \%$} & \multirow{3}{*}{$5.7 \%$} \\
\hline & 11 & 21 & & & & & & \\
\hline & $\begin{array}{c}\mathrm{FN} \\
2\end{array}$ & $\begin{array}{c}\text { VN } \\
191\end{array}$ & & & {$[85,8-94,3 \%]$} & & & \\
\hline
\end{tabular}

S: Sensibilidad E: Especificidad [IC 95\%] Intervalo de confianza 95\% VP: Verdaderos Positivos FP: Falsos Positivos FN: Falsos Negativos VN: Verdaderos Negativos VPP: Valor Predictivo Positivo VPN: Valor Predictivo Negativo P: Prevalencia

Figura 1

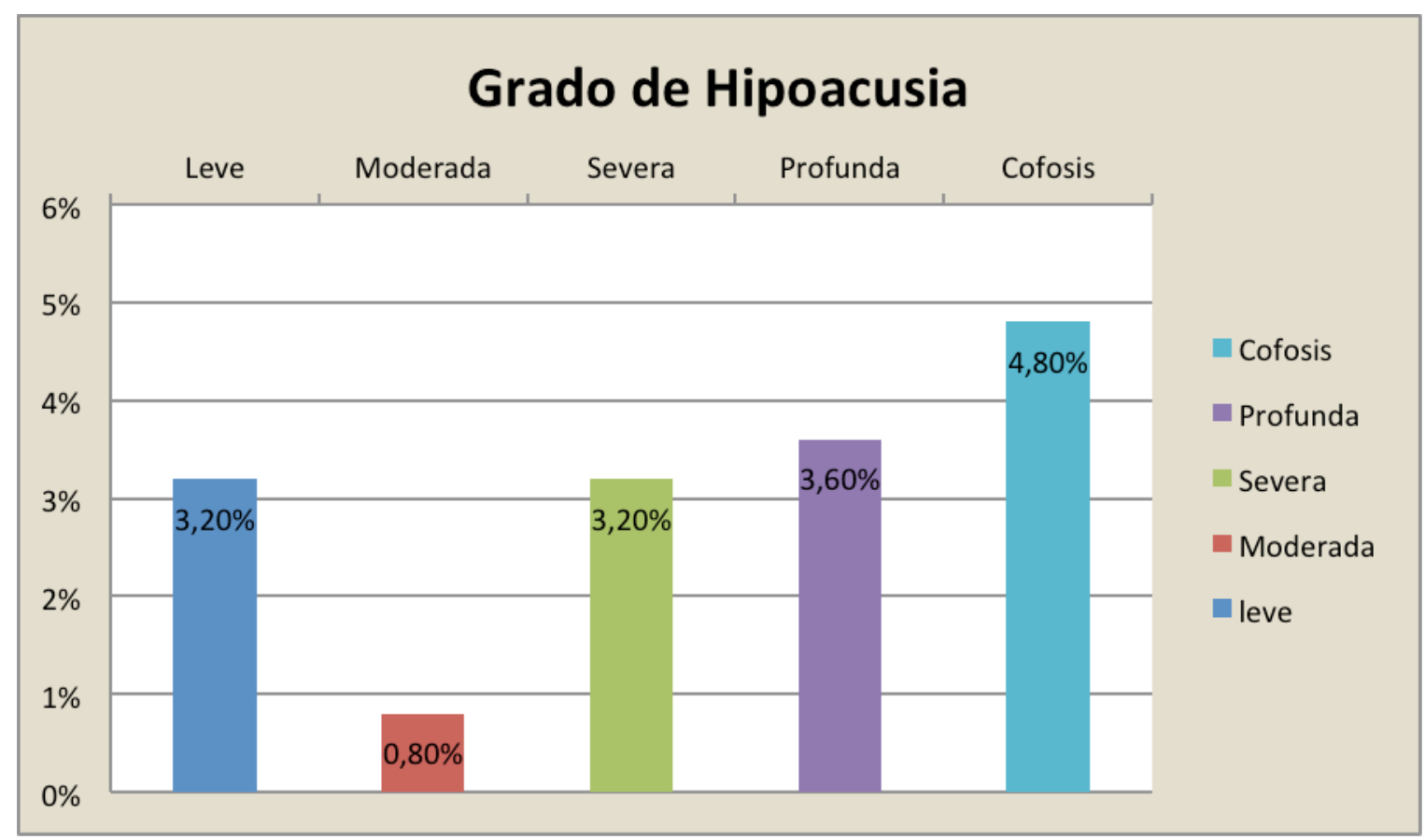

